\title{
Externalized Domestication: Smart Speaker Assistants, Networks and Domestication theory
}

\author{
Saba Rebecca Brause ${ }^{\mathrm{ab}^{*}}$, Grant Blank $\mathrm{c}^{* *}$ \\ ${ }^{a}$ Weizenbaum Institute for the Networked Society, Berlin, Germany \\ ${ }^{b}$ Technical University of Berlin, Berlin, Germany \\ ${ }^{c}$ Oxford Internet Institute, University of Oxford, Oxford, United Kingdom
}

*Saba Rebecca Brause, sbrause@inet.tu-berlin.de, Internet Network Architectures (INET), TU Berlin, MAR 4-4, Marchstr. 23, 10587 Berlin, Germany

** Grant Blank, grant.blank@oii.ox.ac.uk, Oxford Internet Institute, University of Oxford, 1 St Giles', Oxford OX1 3JS, United Kingdom

Word count: 5.996 words (including abstract, table, figure captions, references, footnotes)

Corresponding author: Dr. Grant Blank 


\title{
Externalized Domestication: Smart Speaker Assistants, Networks and Domestication theory
}

\author{
Framed by domestication theory, affordances and use genres, this study explores early \\ adopters' uses of smart speaker assistants (SSAs), like Amazon Echo (Alexa) and \\ Google Home. Based on semi-structured, in-depth interviews, we develop a typology \\ of use genres, and describe spatially distributed uses. The interviews revealed six use \\ genres that go beyond the well-known convenience and entertainment. Specifically, \\ the use genres of companionship, self-control and productivity, sleep aid, health \\ care, peace of mind and increased accessibility emerged from participants' accounts. \\ In addition, we found spatially distributed uses based on the users' perception of the \\ spatial affordances of SSAs. These spatially distributed uses lead us to propose the \\ process of externalization as a necessary extension of domestication theory for the \\ appropriation of networked devices.
}

Keywords: smart speaker assistants, Domestication theory, affordances, Amazon Alexa, Google Home, externalization

\section{Introduction}

The first stand-alone in-home voice assistant devices - "smart speaker assistants" (SSAs) became available in 2014. By 2018, $24 \%$ of US households owned an SSA (Nielsen, 2018), reaching $20 \%$ of UK households by 2019 (Ofcom, 2019). While 'smart speaker' is the most common name for these devices, we use 'smart speaker assistant' to stress the existence of an integrated assistant beyond a connected speaker. SSAs are voice-controlled by the user and smart, meaning they respond to natural language user requests (Hoy, 2018). They come in various shapes and sizes; some models have screens and cameras. Increasingly, SSAs are integrated with other home appliances, allowing people to control thermostats, light bulbs, TVs, security cameras, locks, or sprinklers. Amazon devices have the ability to 'drop in' - or use an Echo device as an intercom to another Echo in an entirely different location, like someone else's house. SSAs can be customized with 'skills' (Amazon) or 'actions' (Google), downloadable from an online store (Hoy, 2016).

Although there have been studies of the form and frequency of users' requests, we draw on 
domestication theory to examine SSA use in greater depth. More specifically, we investigate the research question:

What use genres have been created to domesticate SSAs?

To do so, we develop a typology of use genres (Bakardjieva, 2005, 2006) and describe how spatially dispersed uses of SSAs blur the boundaries of the household. Further, the remote, dispersed, networked affordances of 'smart home' devices are not well captured by the core concepts of domestication theory, which suggests a needed theoretical extension that we call "externalization". We explore the rationale for and implications of this new concept in a separate section below.

This paper is structured in four parts. First, we summarize previous work on SSAs and present our theoretical framework. Second, we describe our methodology. Then we report our findings. Finally, we describe externalization and we conclude with the implications of our findings for the study of the appropriation of always-on networked devices.

\section{Literature review}

Few have studied user experiences with SSAs, and the research is somewhat disconnected. Early work focused on users' experience with smartphone-based, mobile assistants. Kiseleva et al. (2016) identified control of the mobile phone, web searches and structured search dialogues as typical use scenarios, and found high satisfaction levels amongst users. Luger and Sellen (2016) found that convenience and time-saving through the 'hands free' use and the ability to multi-task were key use cases.

Studies of stand-alone in-home SSAs focused on the form and frequency of users' requests towards SSAs. Considering form, Burton and Gaskin (2019) found that people who treat children with politeness also treat digital assistants politely. Porcheron, Fischer, Reeves, and Sharples (2018) showed that commands were embedded amongst everyday family activities and that control over the SSA followed the 'politics of the home' (p.5). Regarding frequency, Bentley et al. (2018) found that the median household performed 4.1 commands per day, and that the most frequent requests were for 'music' (40\%) and 'information' (17\%). Lopatovska et al. (2018) identified information searches, content requests for 'entertainment' - jokes, music or games - and the control of other home devices as the most common requests.

The only investigation of SSA use types so far explored use by people with disabilities (Pradhan, Mehta, and Findlater, 2018). They found that SSAs were easier to use than other devices and improved everyday independence. They found unexpected use cases: speech therapy, learning support and memory support.

Rather than how and when users address their SSAs, we look at what use genres they have developed. We seek to understand the role the devices play in users' lives. Convenience (Luger \& Sellen, 2016) and entertainment (Lopatvska et al., 2018) have been previously identified as use cases - albeit in a mobile context (Luger \& Sellen, 2016) and deduced from content requests (Lopatovska et al., 2018). We therefore emphasize other use cases to understand the variety of ways in which SSAs become meaningful. Similar to work on social media (Hogan \& Quan-Haase, 2010), we focus on uses beyond requests to identify more long-term, stable uses and reach more generalizable claims. 


\title{
Theoretical frameworks: Technology adoption and use
}

\author{
Domestication and the 'moral economy' in the home
}

Domestication theory was developed to describe and analyse how technologies become a part of users' everyday lives, usually in domestic environments (Berker, Hartmann, Punie, \& Ward, 2006). Domestication describes four dimensions of technology appropriation-commodification, objectification, incorporation and conversion (Silverstone, 2006).

Commodification refers to the initial appropriation step - often the purchase - which brings the technology into the home, " "packaged" ...with dreams and fantasies, hopes and anxieties' (Silverstone, 2006, p.234). Such dreams and anxieties are shaped through public representations of the technology, e.g. advertising (du Gay, Hall, Janes, McKay, \& Negus, 2013). Objectification relates to the physical location in the home. Incorporation refers to temporal integration into the domestic life, such as the frequency of use and the development of 'everyday routine(s)' around the technology (Haddon, 2004, pp.139-140). Finally, conversion relinks the domestication process to the outside world, through display, sharing, discussion, and acquiring skills and literacies (Silverstone, 2006).

The domestication of technologies into the home is related to wider socio-technical developments and to the needs and interests of the household. The domestication of technologies is shaped by the users' 'micro-regulation of the medium' (Bakardjieva, 2005, p.138). Micro-regulation refers to decisions in a household about how, when and where to use the technology. It is based on the premise that homes have an alternative 'moral economy', separate from the 'formal or more objective economy' (Silverstone, Hirsch, \& Morley, 1992, p.14). The home moral economy includes a 'domestic culture', which has its own values and interests (p.14). This alternative domestic culture and moral economy provide the basis for appropriating and using the technologies in ways that the formal economy may not originally have intended. Consequently these technologies 'are re-defined in accordance with the household's own values and interests' (Bakardjieva, 2005, p.138).

While domestication originally focused exclusively on the home (Silverstone et al., 1992), recent studies have illustrated how the adoption of new information and communication technologies (ICTs) is leading to a 'renegotiation and re-definition of the boundaries between the (private) home and the public world' (Bakardjieva, 2005, p. 163; cf. de Reuver, Nikou, \& Bouwman, 2016; Green \& Haddon, 2009). We will further argue that domestication theory needs a new concept, externalization, to handle connectable devices.

\section{Use genres}

Developed by Bakardjieva $(2005,2006)$, the concept of 'use genres' is a tool for analysing the similarities and differences in the ways people domesticate a technology. While a use genre is 'contingent upon the inbuilt functionalities' of the technology, it is 'not designer-configured..., rather it emerges out of a concrete practical situation as experienced and defined by a user' (Bakardjieva, 2006, p.73). Thus, the development of use genres is not technologically determined. Instead, users' agency guides them to make the technology effective and meaningful in their specific situations. 


\section{Affordances}

Originally conceptualized in ecological psychology, affordances are what an object offers to a person through its physical properties, but 'measured relative to the' person to whom it affords something (Gibson, 2014, p.120; emphasis in original). An affordance is, therefore, defined both by the physical properties of the environment and perceptions of the participant in that environment.

The concept has found widespread, but inconsistent application in communication technology studies (Nagy \& Neff, 2015; Evans, Pearce, Vitak, \& Treem, 2016). Indeed, technological features, affordances and outcomes from affordances are often insufficiently distinguished in empirical studies of technology use (Evans et al., 2016). Affordances can be 'broadly described as possibilities for action' and are 'the "multifaceted relational structure" (Faraj \& Azad, 2012, p.254), between a technology and the user that enables or constrains potential behavioural outcomes in a particular context' (Evans et al., 2016, p. 36), although outcomes 'need not be an action' (p.49). For example, the listening-responding features of SSAs provide the ability to talk to the device (affordance), to be entertained by hearing a joke (outcome). The affordance lies in the combination of the users' perception and the existence of a feature, the (behavioural) outcome is thus variable (Evans et al., 2016).

Additionally, the translation of a perceived affordance into an outcome also depends on other factors, such as the users' physical or cognitive ability, and the cultural and institutional legitimacy of a behaviour (Davis \& Chouinard, 2016) (see figure 1). For instance, a user may perceive the possibility to control her lights (affordance) through an SSA by connecting the two (feature) but may not do it (no outcome) because she lacks the skills or confidence to connect them.

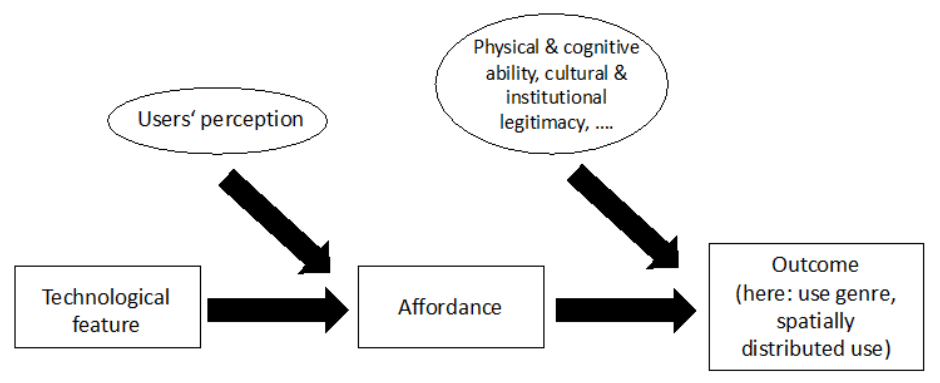

Figure 1 - Our conceptualization of the relationship between affordances and use genres (based on Evans et al., 2016; Davis \& Chouinard, 2016)

In our analysis, we first focus on various use genres without specifically identifying the affordances underlying these behavioural outcomes. In a second part, we concentrate on the spatial affordances of SSAs that enable spatially distributed uses. 


\section{Methods}

Due to the exploratory nature of the study, our interest in individuals' 'life-worlds' (Berg, 2009, p.15) and our research goal to investigate the ways that SSAs become meaningful to users, we used semistructured interviews. Their key strength is their ability to provide in-depth understanding of social behaviour. Interviews 'empower the researcher to probe about facts or about ideal responses or situations, as well as imaginary scenarios and fantasies that simply are not visible in everyday life.' (Lamont \& Swidler, 2014, p.160).

Interview participants were recruited through a mix of opportunistic and snowball sampling (Bryman, 2012). Calls for participation were posted on four Facebook groups of SSA users, two subreddits on Reddit, an Echo user online forum, and one author's twitter account. Interviews took place from June to August 2018 with a total of twelve current users of SSAs; they lasted from 15 to 90 minutes. Interview participants volunteered for the study and we understand the risk of selfselection bias in this recruitment strategy (Lavrakas, 2008). In particular, the sample contained a large number of self-reported technologically proficient users (7 out of 12), which likely impacted the range and technical complexity of uses. Participants' names are pseudonyms. Like other qualitative work, interviewees are not representative of any population. Rather, they are examples of how people use SSAs. We leave the study of use genre frequencies in the population to future survey research. Later interviews tended to be repetitive and added little new knowledge, suggesting that theoretical saturation was reached (Brinkmann \& Kvale, 2015). Interviewees were a mix of ages and genders but demographic variables showed no relation to SSA use, so we will not discuss demographics further.

Interviews were audio-recorded, transcribed and coded with NVivo. The inductive process consisted of identifying themes in the interviews and creating codes for them. The research question and 'use genres' concept provided the main 'analytical objective' that guided the initial development of codes and the coding process, but the analysis within this analytical objective remained inductive, consistent with the exploratory approach of the study (Guest, MacQueen, \& Namey, 2012). We coded as 'use genres' descriptions of how the devices had become meaningful to users in their everyday life, going beyond request types or unrealised action possibilities. Affordances were not an initial interest but they became a second 'analytical objective' at a later stage of analysis as the theme of spatially distributed uses emerged from the data. Themes were analysed on a semantic, explicit level (Braun \& Clarke, 2006, p.84) and identified based on a combination of repetitions, unfamiliar terms or unfamiliar uses of terms, metaphors and analogies, similarities and differences between interview responses, and missing data (Ryan \& Bernard, 2003).

\section{Findings}

\section{Typology of use genres}

From the participants' accounts it became clear that there is no archetypical user, and that they have been developing a variety of use genres related to SSAs beyond the previously known 'convenience' (Luger \& Sellen, 2016) and 'entertainment' (Lopatovska et al., 2018). Companionship, self-control and productivity, sleep aid, health care, peace of mind and increased accessibility of the home emerged as additional use genres. 
First, SSAs were used for companionship in different settings. Susan indicated that she talked to her SSA to avoid loneliness:

...because I live alone, I suppose, I tend to use it as company. I talk to it...just [to] hear a voice in the house...

In contrast, Emily described a dinner party where her device became a 'quasi-other' (Ihde, 2014, p.554) in form of a game master. She explained:

It's quite good for playing "guess that song". But not through a formal app. Just getting it to play a playlist, ...if you're having a dinner party or something, then everyone shouts out - you work out what it is and then you just tell Alexa to play the next song. ...for example I did it with my brother and his girlfriend ...it was quite good because no one was the game master... Everyone could play the game.

Second, to improve self-control and enhance productivity while working at home, Lucas and Emily (respectively) explained that they used their devices' timer:

I often use my Google Home to set ...productivity timers for work.

If I need to concentrate and I set a time for five minutes, and then I have to concentrate for 5 minutes. And then kind of - she, like, keeps me in check.

Here again, especially in the case of Emily, the device becomes a 'quasi-other' that helps the user to stay in control. Timers were also used for meal preparation, e.g. by Michael. But Emily and Lucas's specific use of the timers for productivity was related to the use of the devices in the 'concrete practical situation' (Bakardjieva, 2006, p.73) of working or studying at home. This exemplifies how different 'use genres' can develop from the same technological feature, through different individual perception of an SSA's affordances.

Third, both Emily and Daniel use Alexa skills that help them sleep. While Emily had used 'rain sounds to fall asleep', Daniel explained he had been listening to 'relaxing piano sounds' to sleep.

Fourth, SSAs can aid health care by reminding users to take medication. Peter - a technologically proficient user - created his own private skill "Pill Manager" in order to help him remember when to take pills. He explained:

I'm not in the habit of knowing when to take a pill. But if I do happen to get ill or whatever and I need to go on some sort of medication for a week or two, I have this skill available privately to me. And I can just tell Alexa at what time I took my pill. And then if I forget the next day at what time did I take that pill, I can ask her and she'll tell me back what time I took it. 
Furthermore, SSAs help users find peace of mind when caring for friends and relatives. While Daniel saw great potential in connecting to elderly parents' home (affordance) through the Amazon Alexa's drop-in feature to "check they're okay", he had not (yet) used SSAs that way. However, Peter had set up a SSA in a friend's home in case she needed to call for help:

She is like 89 years old and she's falling frequently now that she's getting older. And I was concerned for her, so I bought her an Echo Dot. And I placed it in the centre of the house, set it up for her. And I'm on her contact list, so if she should fall, she can just ask the echo to call me and let me know that she is in trouble.

Finally, use of an SSA can help them make a home more accessible. Like Pradhan et al.'s (2018) study of disabled users, Peter explained how the devices helped improve a friend's independence.

She's got a lamp next to her chair and it's difficult for her because she is in her 80 s and it's hard for her to reach around and get a hold of the switch to turn the light on. So now she can command the lights on and off with her voice and she's happy with that

Daniel alleviated design constraints to make his home safer.

The house I moved to - all the sockets are in the wrong place. So you walk into the front of the house, and the first light switch was across the first room, which in the winter was bonkers. ...So primarily the rooms I use for voice control is the main sitting room and the main bedroom. And it's just — when you walk through the door, just say "Alexa turn on the lights"

Thus, beyond 'convenience' and 'entertainment' we found six unexpected use genres: companionship, self-control and productivity, health care support, better sleep, peace of mind and improved accessibility (see Table 1). While Pradhan et al. (2018) had already illustrated how the devices could help users with disabilities be more independent, our study showed how this use genre also improved home safety.

[Table 1 about here]

\section{Perceived spatial affordances of SSAs enable spatially distributed uses}

All but three of the interviewees made use of one or more spatial affordances of SSAs to engage in spatially distributed uses. These three spatial affordances consist of (1) the potential ubiquity of SSAs, (2) the link-ability to people inside or outside the home, and (3) the control-ability of home devices. Spatial affordances rely on the use of either several spatially distributed SSAs inside the home or 
between homes, the use of connected home appliances with at least one SSA, or the use of at least one SSA in connection with a phone.

Potential ubiquity: With several devices at home, Lucas described the benefit of having ubiquitous access to the assisting tools of SSAs, for example:

Reminders work really well, I get a notification from my Google Home and a visual notification on my phone. This is why I have a mini in the bathroom for the random shower thoughts I have.

Participants also mentioned the potential ubiquity of audio consumption. For Andrew in-home ubiquitous audio consumption was the central motivation to add SSAs:

I only was going to get one or two. But I found I really, really love listening to music through them because it syncs. ...I do like podcasts, or comedian stuff. I like to listen to words - audiobooks too. So typically, if I leave the room, if I'm doing some going back and forth, this way, I don't miss anything, which is really nice.

Emily described her enthusiasm for seamlessly listening to a song as she moved from outside into her home while switching from her smartphone to an SSA:

I'd be commuting home listening to my music, ...I'd be playing a really good song as I walk in the door, and I didn't want to stop the music. But the ability to look on my phone, go onto Spotify and just flip from my headphones onto the Echo immediately and continue that same song in my living room ...this just brought me an immense amount of joy.

Link-ability, the second spatial affordance, connects spatially separated people. For example, we saw that Peter placed an SSA in a friend's home so that she could call him in an emergency (see 'peace of mind' use genre) that Daniel pondered using the 'drop-in' feature to check in on elderly relatives, linking the people in two different homes. Peter also used this affordance inside the same home, e.g. to communicate with his wife through the 'announce' feature:

So for example if I don't know for sure where my wife is - it's not that big of a house, but we do get lost, sometimes, if I'm getting ready to leave the house, I will just say "do an announce" and it will play that announcement with my voice on it throughout all the echo devices in the home. So I can let her know that I'm leaving and she knows and I don't have to worry about "did she hear?". Because we have Echos in every room.

Peter and his wife also use a grocery list 'skill'; they live-updated shopping lists while one partner was at home and the other was shopping, linking the user inside the home with the partner outside via the SSA and a smartphone: 
I can be on my way to the grocery store and my wife thinks of something else, then she can add it to the list while I'm on the way to the store.

Finally, control-ability allows users with an SSA to control connected devices. Adam described how his 'good night, house' command allows him to control devices throughout his home:

It's something that we do every single night and have done for about a year. We always say - have to say "good night house" and it switches things off, and ...it switches off lights outside, lights inside. ... So there's a lot of stuff that switches off through there, to make sure we haven't left anything on. It switches off amplifiers. It stops things from streaming off the internet ... And it switches on the lights upstairs in the bedrooms.

\section{Externalization: An expansion of Domestication Theory}

This analysis shows that, like earlier ICTs - the Internet (Bakardjieva, 2005), mobile phones (Green \& Haddon, 2009), smartphones (de Reuver et al., 2016) - the use of SSAs re-defines and renegotiates the boundaries between the private home and outside world. Specifically, the link-ability and potential ubiquity affordances of SSAs allow spatially distributed uses that blur these boundaries; for example, through the use of a collective shopping list or when a user can switch music from her smartphone to the SSA when entering her home.

This theme also suggests additional complexity in the domestication of SSAs. When uses are spatially distributed the domestication of an SSA impacts the domestication of other devices. All four domestication processes are involved. For instance, users may decide on the physical placement (objectification) in relation to the other devices that the SSA is connected to. If a user wants to be able to dictate reminders anywhere in the home (e.g. 'random shower thoughts'), there must be a listening SSA in every room. In addition, if a user uses an SSA to switch off all the devices in the house at bedtime, the control-ability affordance (perceived SSA ability to control other devices) adds devices to a nighttime-routine (incorporation) (e.g. Adam's 'good night house' custom-made command). Furthermore, this networked nature can be the reason for users to acquire SSAs (commodification), as in the case of Andrew who bought more SSAs than he originally intended to enable ubiquitous podcast consumption. As another example, Daniel said his original intention in acquiring SSAs was to add voice-control to existing home automation devices. Finally, users may also give SSAs to friends and family (conversion), for voice-based emergency calls (Peter's 'peace of mind' use genre).

Thus, the spatially distributed, network abilities of SSAs influence all four domestication processes. Through this mechanism the domestication of an SSA influences the domestication of any other device that it can connect to, including remotely across households. The four core concepts of domestication theory do not sensitize researchers to the ways that multiple, interacting, spatially distributed devices are domesticated differently than prior devices like mobile phones or televisions. The ability to remotely connect other households to a domestic network adds further complexity as this increases the number of devices and people involved in domestication decisions. Therefore the analytical framework of domestication theory needs expansion to focus theoretical and empirical 
attention on the implications of the networked, dispersed, always-on and possibly remote characteristics of connected home technologies.

We propose to call this process "externalization", defined as the impact of networked devices in each other's domestication. But externalization is itself influenced by the moral economy and domestic culture with its own values and interests - as in the case of the user who set up a remote SSA in a friend's home so she could make emergency calls.

\section{Discussion}

SSAs are promoted as smart helpers that make users' lives easier. The goal of this paper was to explore how users actually used their device. Framed by domestication theory (Silverstone et al., 1992), use genres (Bakardjieva, 2005, 2006) and affordances (Gibson, 2014), we proposed a typology of eight use genres and discussed spatially distributed uses of SSAs.

Interviewees described use genres that go beyond 'convenience' and 'entertainment'. New use genres identified in this study are companionship, self-control and productivity, health care support, better sleep, peace of mind and improved accessibility. While Pradhan et al. (2018) already illustrated how these devices could improve disabled users' independence, our study showed how this use genre also included home safety improvements. However, new use genres can still develop. Indeed, after submission we found a news account of a husband using SSAs and connected devices to remotely spy on his wife at home (Dickinson, 2019).

As they invented use genres, users redefined some features of SSAs. For example, voicecontrol of playlists as a game master illustrates the influence of the moral economy and domestic culture in SSA appropriation. While the manufacturers of SSAs, the music producers and the music platform providers likely had not intended this use case, this user and her guests demonstrated their agency by redefining a feature 'in accordance with the household's own values and interests' (Bakardjieva, 2005, p. 138).

The implication of SSAs as 'quasi-other' in the companionship use genre, and in Emily's account of the self-control/productivity use genre ('she ...keeps me in check'), shows three ways in which users may enter into 'alterity relations' (Ihde, 2014, pp.553-559) with SSAs. Alterity relations are a form of human-technology relations which are characterised as 'relations to or with a technology' (p.553). In three instances, users stand in an alterity relation with their SSAs: a companion for conversation, a game master and an 'other' helping users focus on work.

The theme of spatial affordances emerged from the interview data; not from our starting framework. These spatial affordances consist of the potential ubiquity of SSA uses, the link-ability of people inside and outside the home, as well as the control-ability over other devices.

The implications of the spatially distributed uses are twofold. First, like earlier ICTs (Internet, mobile phone, smartphone), the spatially distributed uses of SSAs reconfirm the re-definition and renegotiation of the boundaries between the private home and the outside world. Second, spatially distributed uses suggest an addition to the domestication framework: voice-controlled technologies in the connected home may be part of a network of technologies. Yet, the domestication of one networked device can impact the domestication of another and vice-versa. The framework thus needs to take into account the potentially networked nature of the domestication process. We have called this process "externalization" and defined it as the impact of networked technologies on each other's domestication. 
SSAs are different from other communication devices. Although previous ICTs, especially mobile phones, provide users with the spatial affordance of link-ability (of people), SSAs distinguish themselves in particular through their potential to connect with other home appliances (controlability). SSAs control other devices and phones don't (exception: smartphones with a mobile assistant). In contrast to a mobile phone assistant, SSAs can become ubiquitous inside homes (potential ubiquity) and link one user with another (link-ability) without either user carrying a device and manually activating it. The spatial affordances of in-home SSAs thus stem from their always-on networked nature. This creates a new opportunity for the moral economy of the household to assert itself. Depending on their values a household may choose to use an SSA to automate garden work or select TV channels, or for monitoring children or emergency communication. Depending on issues like cost, convenience, skills, and values a household could choose to automate none of these or all four.

Future studies of the appropriation of connected devices will therefore need to take the potentially networked, always-on nature of ICTs into account to illuminate how the appropriation of a device fits within an ecosystem of connected devices. SSAs are the entering wedge of the 'smart home' where multiple devices connect and communicate with each other. Device connection may become ubiquitous and this will influence domestication of many devices.

Due to space constraints, we omitted discussing the challenges of SSAs. We enumerate three dimensions of problems here to avoid overstating the devices' usefulness and convenience. On the user side, low technological proficiency and the lack of information about device set-up and capabilities form barriers to SSA use. On the device level, the unreliability of the device and some of its features, the unintended activation of the device, the lack of contextual understanding and the lack of coordinated control emerged from interviewees' accounts. On the infrastructural level, participants mentioned the lack of stable Internet connectivity, the physical structure of the home, the lack of interoperability between proprietary systems, and the cost of connectable streaming services as limitations. These problems mean that SSAs are not easy to install, fully reliable, and always usable. Future research could explore these issues.

Despite their relatively rapid take-up, SSAs still pose a range of challenges, from ease- of-use, security, and the acquisition of new technological literacies to the potential re-negotiation of privacy in a space that has traditionally been considered as private - the home. 


\section{Bibliography}

Bakardjieva, M. (2005). Internet society: The Internet in everyday life. London: SAGE.

Bakardjieva, M. (2006). Domestication running wild. From the moral economy of the household to the mores of a culture. In T. Berker, M. Hartmann, Y. Punie, \& K. Ward (Eds.), Domestication of Media and Technology (pp. 62-79). Berkshire: McGraw-Hill Education.

Bentley, F., Luvogt, C., Silverman, M., Wirasinghe, R., White, B., \& Lottrjdge, D. (2018, September). Understanding the long-term use of smart speaker assistants. Proceedings of the ACM on Interactive, Mobile, Wearable and Ubiquitous Technologies (pp. 91:1-91:24). New York: ACM.

Berg, B. L. (2009). Qualitative research methods for the social sciences (7th ed.). Boston; London: Allyn \& Bacon.

Berker, T., Hartmann, M., Punie, Y., \& Ward, K. (2006). Domestication of Media and Technology. Berkshire: Open University Press.

Braun, V., \& Clarke, V. (2006). Using thematic analysis in psychology. Qualitative Research in Psychology, 3(2), 77-101.

Brinkmann, S., \& Kvale, S. (2015). InterViews: Learning the craft of qualitative research interviewing (3rd ed.). Los Angeles: Sage.

Bryman, A. (2012). Social research methods (4th ed.). Oxford: Oxford University Press.

Burton, N., \& Gaskin, J. (2019, August). "Thank You, Siri": Politeness and intelligent digital assistants. AMCIS 2019 Proceedings (pp. 1-10). Atlanta, GA: AIS.

Davis, J. L., \& Chouinard, J. B. (2016). Theorizing affordances: From request to refuse. Bulletin of Science, Technology \& Society, 36(4), 241-248.

De Reuver, M., Nikou, S., \& Bouwman, H. (2016). Domestication of smartphones and mobile applications: A quantitative mixed-method study. Mobile Media \& Communication, 4(3), 347-370.

Dickinson, A. (2019, September 7). Smart home turns husband into big brother. Washington Post. Retrieved from https://www.washingtonpost.com/

Du Gay, P., Hall, S., Janes, L., McKay, H., \& Negus, K. (2013). Doing cultural studies: The story of the Sony Walkman (2nd ed.). London: SAGE.

Evans, S. K., Pearce, K. E., Vitak, J., \& Treem, J. W. (2016). Explicating affordances: A conceptual framework for understanding affordances in communication research. Journal of Computer-Mediated Communication, 22(1), 35-52. 
Faraj, S., \& Azad, B. (2012). The materiality of technology: An affordance perspective. In P. M. Leonardi, B. A. Nardi, \& J. Kallinikos (Eds.), The Materiality of Technology: An Affordance Perspective (pp. 237-258). Oxford, England: Oxford University Press.

Gibson, J. J. (2014). The ecological approach to visual perception: Classic edition. Retrieved from https://ezproxy-prd.bodleian.ox.ac.uk/login?url= http://ebookcentral.proquest.com/lib/oxford/detail.action?docID=1864844

Green, N., \& Haddon, L. (2009). Mobile communications: An introduction to new media. Oxford: Berg.

Guest, G., MacQueen, K. M., \& Namey, E. E. (2012). Applied thematic analysis. Retrieved from https://ezproxy-prd.bodleian.ox.ac.uk/login?url= http://methods.sagepub.com/book/applied-thematic-analysis

Haddon, L. (2004). Information and communication technologies in everyday life: A concise introduction and research guide. Oxford: Berg.

Hogan, B., \& Quan-Haase, A. (2010). Persistence and change in social media. Bulletin of Science, Technology \& Society, 30(5), 309-315.

Hoy, M. B. (2018). Alexa, Siri, Cortana, and more: An introduction to voice assistants. Medical Reference Services Quarterly, 37(1), 81-88.

Ihde, D. (2014). A phenomenology of technics. In R. C. Scharff \& V. Dusek (Eds.), Philosophy of technology: The technological condition: an anthology (2nd ed., pp. 539-560). Chichester, England : Wiley.

Kiseleva, J., Williams, K., Hassan Awadallah, A., Crook, A., Zitouni, I., \& Anastasakos, T. (2016). Predicting user satisfaction with intelligent assistants. Proceedings of the 39th International ACM SIGIR Conference on Research and Development in Information Retrieval (pp. 45-54). New York: ACM.

Lamont, M., \& Swidler, A. (2014). Methodological pluralism and the possibilities and limits of interviewing. Qualitative Sociology, 37(2), 153-171.

Lavrakas, P. J. (2008). Self-selection bias. Retrieved from SAGE Research Methods: https://methods.sagepub.com/reference/encyclopedia-of-survey-researchmethods/n526.xml.

Lopatovska, I., Rink, K., Knight, I., Raines, K., Cosenza, K., Williams, H., Martinez, A. (2018). Talk to me: Exploring user interactions with the Amazon Alexa. Journal of Librarianship and Information Science, 51(4), 984-997. 
Luger, E., \& Sellen, A. (2016). "Like having a really bad PA": The gulf between user expectation and experience of conversational agents. Proceedings of the $2016 \mathrm{CHI}$ Conference on Human Factors in Computing Systems (pp. 5286-5297).

Nagy, P., \& Neff, G. (2015). Imagined affordance: Reconstructing a keyword for communication theory. Social Media + Society, 1(2), 1-9.

Nielsen. (2018, September 27). (Smart) Speaking my language: Despite their vast capabilities, smart speakers are all about the music. Retrieved from https://www.nielsen.com/us/en/insights/article/2018/smart-speaking-my-languagedespite-their-vast-capabilities-smart-speakers-all-about-the-music

Ofcom. (2019). Media nations: UK 2019 (Report No.2) Retrieved from https://www.ofcom.org.uk/ data/assets/pdf file/0019/160714/media-nations-2019-ukreport.pdf

Porcheron, M., Fischer, J. E., Reeves, S., \& Sharples, S. (2018). Voice Interfaces in Everyday Life. Proceedings of the 2018 CHI Conference on Human Factors in Computing Systems (pp. 640:1-640:12). New York: ACM.

Pradhan, A., Mehta, K., \& Findlater, L. (2018). Accessibility came by accident: Use of voicecontrolled intelligent personal assistants by people with disabilities. Proceedings of the 2018 CHI Conference on Human Factors in Computing Systems (pp. 459:1-459:13). New York: ACM.

Ryan, G. W., \& Bernard, H. R. (2003). Techniques to identify themes. Field Methods, 15(1), 85109.

Silverstone, R. (2006). Domesticating domestication. In T. Berker, Y. Punie, M. Hartmann, \& K. Ward (Eds.), Domestication of Media and Technology (pp. 229-248). Berkshire: McGrawHill Education.

Silverstone, R., Hirsch, E., \& Morley, D. (1994). Information and communication technologies and the moral economy of the household. In R. Silverstone \& E. Hirsch (Eds.), Consuming technologies. Media and information in domestic spaces. (pp. 13-27). London: Routledge. 